\title{
Superconducting nanofilms: Andreev-type states induced by quantum confinement
}

\author{
A. A. Shanenko, ${ }^{1}$ M. D. Croitoru, ${ }^{2}$ and F. M. Peeters ${ }^{1}$ \\ ${ }^{1}$ TGM, Departement Fysica, Universiteit Antwerpen, Groenenborgerlaan 171, B-2020 Antwerpen, Belgium \\ ${ }^{2}$ EMAT, Departement Fysica, Universiteit Antwerpen, Groenenborgerlaan 171, B-2020 Antwerpen, Belgium
}

(Received 21 April 2008; published 11 August 2008)

\begin{abstract}
Quantum confinement of the transverse electron motion is the major effect governing the superconducting properties of high-quality metallic nanofilms, leading to a nonuniform transverse distribution of the superconducting condensate. In this case the order parameter can exhibit significant local enhancements due to these quantum-size effects and, consequently, quasiparticles have lower energies when they avoid the local enhancements of the pair condensate. Such excitations can be considered as new Andreev-type quasiparticles but now induced by quantum confinement. By numerically solving the Bogoliubov-de Gennes equations and using Anderson's approximate solution to these equations, we: (a) formulate a criterion for such new Andreev-type states (NATS) and (b) study their effect on the superconducting characteristics in metallic nanofilms. We also argue that nanofilms made of low-carrier-density materials, e.g., of superconducting semiconductors, can be a more optimal choice for the observations of NATS and other quantum-size superconducting effects.
\end{abstract}

DOI: 10.1103/PhysRevB.78.054505

PACS number(s): 74.78.Na

\section{INTRODUCTION}

Well-controlled downsizing and shaping of nanostructures below the $10-20 \mathrm{~nm}$ scale have become recently possible due to a sustainable advancement in nanofabrication technologies. It is expected that in the near future, one will be able to routinely control surface features as small as $1 \mathrm{~nm} .^{1}$ Another impressive achievement is that the reduction in sample size is accompanied by a progressive improvement in quality of micro- and nano-objects. Recently, several experimental groups have reported the fabrication of high-quality metallic nanostructures with minimal disorder. With today's technology, we have at our disposal single-crystal $\mathrm{Pb}$ atomically uniform films with thickness down to a few monolayers (ML) ${ }^{2-4}$ single-crystalline Sn nanowires with width of about $20-40 \mathrm{~nm},{ }^{5,6}$ and polycrystalline $\mathrm{Al}$ nanowires (with practically no tunnel barrier between grains) with diameters down to and even below $10 \mathrm{~nm}^{7}$

The electron mean-free path in high-quality nanofilms and nanowires was estimated to be about or larger than the specimen thickness. ${ }^{4,7}$ The photoemission spectra of such singlecrystal $\mathrm{Pb}$ nanofilms exhibited clear signatures of a splitting of the electron band into a series of subbands due to the transverse quantization of the electron motion..$^{2}$ In addition, even extremely thin single-crystal metallic films, with thickness of about 5-7 ML, show no significant indications of defect- or phase-driven suppression of superconductivity (see Ref. 3 and discussion in the second paper given in Ref. 4). The same is true for the high-quality nanowires [except for the most narrow (with width of 6-8 $\mathrm{nm}$ ) and very long (with length of $10-50 \mu \mathrm{m}$ ) aluminum samples ${ }^{7,8}$. Thus, we may conclude that the so-called Anderson theorem ${ }^{9}$ controls the effect of elastic scattering on inevitable nonmagnetic impurities in this case. Recall that the exact formulation of this theorem reads "within the linear approximation in the impurity density of the superconducting quantities are not dependent on $\tau^{-1}$, the rate of the elastic impurity scattering." 10

Most of the previous theoretical studies deal with superconductivity in strongly disordered or granular nanosystems.
In such cases the Anderson theorem is violated due to the enhancement of the Coulomb repulsion ${ }^{11}$ and because of mesoscopic fluctuations ${ }^{12}$ (see discussion in Ref. 13). For diffusive electron motion, the Coulomb-interaction effects lead to a suppression of the critical temperature with increasing disorder. ${ }^{14}$ Mesoscopic fluctuations can result in superconducting islands ${ }^{13}$ instead of a uniform superconducting condensate. In the present investigation, we are focused on metallic nanofilms with minimal disorder, where the Coulombinteraction effects and mesoscopic fluctuations are not of crucial importance. The major mechanism governing superconductivity in this nearly clean regime is the quantum-size confinement of electrons.

Since the classical works by Bogoliubov ${ }^{15}$ and Gor'kov, ${ }^{16}$ it is well established that the superconducting order parameter $\Delta(\mathbf{r})$ can be treated as a wave function for the center-ofmass motion of the Cooper pairs. Then, when the transverse quantization of the electron spectrum in films becomes important, one expects a nonuniform transverse distribution of the order parameter (at least, at minimal disorder). From numerical investigations of the Bogoliubov-de Gennes (BdG) equations, it was found that below thicknesses of about 10 $\mathrm{nm}$, such variations are significantly enhanced due to quantum-size effects. ${ }^{17}$ As known, spatial variations of $\Delta(\mathbf{r})$ can result in Andreev reflection and quantization of lowenergy excitations. ${ }^{18,19}$ To some extent, quasiparticles feel a spatial variation of the absolute value of the superconducting order parameter as a potential barrier [Andreev mechanism (AM)]. The nonuniform spatial distribution of the order parameter induced by quantum confinement gives rise to the formation of new Andreev-type states (NATS). Contrary to the usual Andreev states, NATS cannot be completely localized in domains where the order parameter is suppressed due to the fact that the specimen thickness is much less than the bulk coherence length. Recently, we found for a clean metallic nanocylinder that the formation of NATS can strongly reduce the ratio of the excitation energy gap to the critical temperature..$^{20}$ The present paper is aimed to generalize our previous study to the case of superconducting nanofilms. 
Our paper is organized as follows: In Sec. II we outline the formalism of the BdG equations for films and then discuss Anderson's approximate solution of these equations. Such a solution simplifies the analysis of our numerical results and makes it possible to formulate a criterion for NATS. In Sec. III, based on a numerical self-consistent solution of the BdG equations, we investigate NATS in clean metallic films with a uniform thickness of $\lesssim 10 \mathrm{~nm}$. Then, in Sec. IV, we discuss the possibility of observing NATS and argue that nanofilms made of low-carrier-density materials, e.g., superconducting semiconductors, can be a more optimal variant for experimental investigations of NATS together with other quantum-size superconducting effects.

\section{FORMALISM AND NATS CRITERION}

\section{A. Bogoliubov-de Gennes equations}

The transverse quantization of the electron motion in a clean film results in a superconducting order parameter that depends on the transverse spatial coordinate. To investigate the equilibrium superconducting properties of such a system, one should solve the $\mathrm{BdG}$ equations ${ }^{21}$ rather than the Ginzburg-Landau equations suitable for the mesoscopic and bulk regimes. The $\mathrm{BdG}$ equations can be written as

$$
\begin{gathered}
E_{i}\left|u_{i}\right\rangle=\hat{H}\left|u_{i}\right\rangle+\hat{\Delta}\left|v_{i}\right\rangle, \\
E_{i}\left|v_{i}\right\rangle=\hat{\Delta}^{*}\left|u_{i}\right\rangle-\hat{H}^{*}\left|v_{i}\right\rangle,
\end{gathered}
$$

where $E_{i}$ is the excitation (quasiparticle) energy and $u_{i}(\mathbf{r})$ $=\left\langle\mathbf{r} \mid u_{i}\right\rangle$ and $v_{i}(\mathbf{r})=\left\langle\mathbf{r} \mid v_{i}\right\rangle$ are the particlelike and holelike wave functions. The single-electron Hamiltonian in Eqs. (1a) and (1b) is given by

$$
\hat{H}=\frac{1}{2 m}\left(\hat{\mathbf{p}}-\frac{e}{c} \mathbf{A}\right)^{2}+V_{\text {conf }}(\hat{\mathbf{r}})-E_{F},
$$

where $E_{F}$ is the Fermi level, $m$ is the electron band mass (set to the free-electron mass $m_{e}$ below), and $V_{\text {conf }}(\hat{\mathbf{r}})$ stands for the confinement potential. The matrix element of $\left\langle\mathbf{r}|\hat{\Delta}| \mathbf{r}^{\prime}\right\rangle$ is directly related to the superconducting order parameter by

$$
\left\langle\mathbf{r}|\hat{\Delta}| \mathbf{r}^{\prime}\right\rangle=\Delta(\mathbf{r}) \delta\left(\mathbf{r}-\mathbf{r}^{\prime}\right),
$$

with $\delta(x)$ as the Dirac $\delta$ function. The BdG equations are solved in a self-consistent manner together with the relation

$$
\Delta(\mathbf{r})=g \sum_{i} u_{i}(\mathbf{r}) v_{i}^{*}(\mathbf{r})\left(1-2 f_{i}\right),
$$

with $g$ as the coupling constant and $f_{i}=1 /\left(e^{\beta E_{i}}+1\right)$ [here $\beta$ $=1 /\left(k_{B} T\right)$ with $T$ as the temperature and $k_{B}$ as the Boltzmann constant]. The sum in Eq. (4) is restricted to the states with positive excitation energies (below we set $\mathbf{A}=0$ ) and with the single-electron energy (measured from the Fermi level),

$$
\xi_{i}=\left\langle u_{i}|\hat{H}| u_{i}\right\rangle+\left\langle v_{i}|\hat{H}| v_{i}\right\rangle \in\left[-\hbar \omega_{D}, \hbar \omega_{D}\right],
$$

within the Debye window with $\omega_{D}$ as the Debye frequency.

We assume that the confinement potential $V_{\text {conf }}(\mathbf{r})$ is zero inside the sample and infinite outside. This results in the quantum confinement boundary conditions for the transverse $z$ direction

$$
\left.u_{i}\right|_{z=0}=\left.u_{i}\right|_{z=d}=0,\left.\quad v_{i}\right|_{z=0}=\left.v_{i}\right|_{z=d}=0,
$$

with $d$ as the film thickness. In the longitudinal $x$ and $y$ directions, one can use periodic boundary conditions. In this case $\Delta(\mathbf{r})=\Delta(z)$ and

$$
u_{i}(\mathbf{r})=\frac{e^{\imath k_{x} x}}{\sqrt{L_{x}}} \frac{e^{i k_{y} y}}{\sqrt{L_{y}}} u_{n}(z), \quad v_{i}(\mathbf{r})=\frac{e^{\imath k_{x} x}}{\sqrt{L_{x}}} \frac{e^{i k_{y} y}}{\sqrt{L_{y}}} v_{n}(z),
$$

with $i=\left\{k_{x}, k_{y}, n\right\}$, where $k_{x}$ and $k_{y}$ are the wave numbers controlling the electron quasifree motion parallel to the film and $n=0,1,2 \ldots$ is the number of nodes of the transverse wave functions $u_{n}(z)$ and $v_{n}(z)$. Note that when $\Delta(z) \neq 0$, $u_{n}(z)$ and $v_{n}(z)$ are not the solutions of the one-dimensional Schrödinger equation for a particle confined in the domain $0<z<d$.

To numerically solve the BdG equations, we use the following procedure: First, we expand $u_{i}(\mathbf{r})$ and $v_{i}(\mathbf{r})$ in terms of the eigenfunctions of $\hat{H}$ and so convert Eqs. (1a) and (1b) into a matrix equation. Then, the numerical problem is solved by means of diagonalizing the relevant matrix and invoking iterations in order to account for the selfconsistency relation given by Eq. (4). More details can be found in Ref. 17.

\section{B. Anderson's approximate solution}

In Sec. II A we outlined the formalism of the BdG equations for a clean superconducting nanofilm. In particular, it was mentioned that the particlelike and holelike wave functions are not the eigenfunctions of $\hat{H}$ in the presence of superconducting order. However, as it follows from our numerical investigations, they are close to each other within the accuracy of less than a few percent for $d \lesssim 10 \mathrm{~nm}$. So, we can use Anderson's approximate solution ${ }^{9}$ in order to obtain analytical expressions useful to interpret our numerical results. In the absence of a magnetic field and a supercurrent, Anderson's "recipe" reads

$$
\left|u_{i}\right\rangle=U_{i}|i\rangle, \quad\left|v_{i}\right\rangle=V_{i}|i\rangle, \quad \hat{H}|i\rangle=\hat{H}^{*}|i\rangle=\xi_{i}|i\rangle,
$$

where

$$
\langle\mathbf{r} \mid i\rangle=\frac{e^{\imath k_{x} x}}{\sqrt{L_{x}}} \frac{e^{\imath k_{y} y}}{\sqrt{L_{y}}} \varphi_{n}(z), \quad \varphi_{n}(z)=\sqrt{\frac{2}{d}} \sin \left[\frac{\pi(n+1) z}{d}\right],
$$

and $|i\rangle$ stands for $\left|k_{x}, k_{y}, n\right\rangle$. Now, inserting Eq. (8) into Eqs. (1a) and (1b), one gets

$$
\begin{aligned}
& E_{i} U_{i}=\xi_{i} U_{i}+\Delta_{i} V_{i}, \\
& E_{i} V_{i}=\Delta_{i}^{*} U_{i}-\xi_{i} V_{i},
\end{aligned}
$$

with $\Delta_{i}=\langle i|\hat{\Delta}| i\rangle$ and 


$$
\xi_{i}=\frac{\hbar^{2} k_{x}^{2}}{2 m}+\frac{\hbar^{2} k_{y}^{2}}{2 m}+\frac{\hbar^{2}}{2 m} \frac{\pi^{2}(n+1)^{2}}{d^{2}} .
$$

Equations (9a) and (9b) have a nontrivial solution only when $E_{i}= \pm \sqrt{\xi_{i}^{2}+\left|\Delta_{i}\right|^{2}}$. The physical (stable) solution is the one with the plus sign and so we find

$$
E_{i}=\sqrt{\xi_{i}^{2}+\left|\Delta_{i}\right|^{2}}, \quad U_{i} V_{i}^{*}=\frac{\Delta_{i}}{2 E_{i}},
$$

where the normalization condition

$$
\left|U_{i}\right|^{2}+\left|V_{i}\right|^{2}=1
$$

is used. Note that as seen from Eq. (8), $\Delta_{i}$ does not depend on $k_{x}$ and $k_{y}$ and so

$$
\Delta_{i}=\Delta_{n}=\int_{0}^{d} d z \varphi_{n}^{2}(z) \Delta(z)
$$

Equations (10) and (12) make it possible to recast the selfconsistency relation (4) in the form of a BCS-type gap equation. At $T=0$, for instance, it reads

$$
\Delta_{n}=-\sum_{i^{\prime}} \frac{V_{n n^{\prime}} \Delta_{n^{\prime}}}{2 \sqrt{\xi_{i^{\prime}}+\left|\Delta_{n^{\prime}}\right|^{2}}},
$$

with the pair-interaction matrix element given by

$$
V_{n n^{\prime}}=-\frac{g}{L_{x} L_{y}} \int_{0}^{d} d z \varphi_{n}^{2}(z) \varphi_{n^{\prime}}^{2}(z) .
$$

Solving Eq. (13), one can find Anderson's approximate solution of the BdG equations. Why is it approximate? In addition to Eqs. (9a) and (9b), we obtain from the BdG equations $\left\langle i^{\prime}|\hat{\Delta}| i\right\rangle=0$ for $i \neq i^{\prime}$, which is not always compatible with Eqs. (9a) and (9b) and, hence, Eq. (13). Note that Eq. (13) can also be derived within the multiband BCS approximation (see, for example, Ref. 22) governed by the Hamiltonian

$$
\hat{H}_{\mathrm{mult}}=\sum_{i \sigma} \xi_{i} a_{i \sigma}^{\dagger} a_{i \sigma}+\sum_{i i^{\prime} \sigma} \frac{V_{n n^{\prime}}}{2} a_{i \sigma}^{\dagger} a_{i-\sigma}^{\dagger} a_{\bar{i}^{\prime}-\sigma}^{\dagger} a_{i^{\prime} \sigma},
$$

where $a_{i \sigma}^{\dagger}\left(a_{i \sigma}\right)$ are the creation (annihilation) operators for a fermion in the state $(i, \sigma)$ with $\sigma$ as the electron-spin projection and $\bar{i}=\left\{-k_{x},-k_{y}, n\right\}$. The main assumption of the multiband BCS approach is the neglect of the electron pairing between different subbands, i.e., $\left\langle a_{i \sigma} a_{i^{\prime}-\sigma}\right\rangle=0$. One can expect, and this is supported by our numerical results, that Eq. (8) is a good approximation when

$$
\frac{\hbar^{2}}{2 m} \frac{\pi^{2}}{d^{2}}>\Delta_{n} \quad(\forall n),
$$

with $\Delta_{n}$ given by Eq. (12), i.e., when the transverse quantization plays a significant role.

\section{Criterion for NATS}

Now we have everything at our disposal to formulate a criterion that determines which quasiparticles are successive in avoiding local enhancements of the superconducting order parameter. This is of importance because NATS are not completely localized in the domains of a suppressed order parameter. The characteristic scale of the transverse spatial variations of the pair condensate in nanofilms is much less than the bulk coherence length, ${ }^{17}$ and so we cannot expect the formation of bound states similar to Andreev states localized in the core of an isolated vortex. Hence, to explore effects of $\mathrm{AM}$ on the nanoscale, we need to invoke another criterion. As mentioned in Sec. I, AM implies that quasiparticles "feel" a spatial variation of the absolute value of the order parameter as a kind of potential barrier. Therefore, it is reasonable to work with the mean value of $|\Delta(\mathbf{r})|$ that is seen by the quasiparticles of a given sort, i.e.,

$$
\langle|\Delta(\mathbf{r})|\rangle_{i}=\int d^{3} r|\Delta(\mathbf{r})|\left[\left|u_{i}(\mathbf{r})\right|^{2}+\left|v_{i}(\mathbf{r})\right|^{2}\right],
$$

where the quasiparticle spatial distribution $\left|u_{i}(\mathbf{r})\right|^{2}+\left|v_{i}(\mathbf{r})\right|^{2}$ is a normalized function due to Eq. (11). What is the link between $\langle|\Delta(\mathbf{r})|\rangle_{i}$ and the quasiparticle energy $E_{i}$ given by Eq. (10)? Using Eqs. (8) and (11), one can get

$$
\left|\Delta_{i}\right|=\langle|\Delta(\mathbf{r})|\rangle_{i}
$$

The simplest way to prove Eq. (17) is to choose the order parameter as a real and non-negative quantity, which can always be done in the absence of a magnetic field and a supercurrent.

Equation (17) makes it possible to conclude that $\mathrm{AM}$ manifests itself through the formation of the multigap structure $\left(\Delta_{n} \neq \Delta_{n^{\prime}}\right)$ and NATS are specified by the minimal value of $\left|\Delta_{n}\right|$. It is worth noting that one should be careful with the word "gap" often used for $\Delta_{n}$ within the multiband BCS approach. The point is that even in the absence of a supercurrent and a magnetic field, $\Delta_{n}$ can be treated as the energy gap for quasiparticles of the $n$ branch only when the bottom of the corresponding single-electron subband $\hbar^{2} \pi^{2}(n$ $+1)^{2} /\left(2 m d^{2}\right)$ is below the Fermi level. This is mostly the case for quasiparticles contributing to the order parameter [whose single-electron energies are located in the Debye window, see Eq. (5)]. However, the single-electron subbands move in energy with changing $d$ and there are situations when the bottom of a single-electron subband is in the Debye window but above the Fermi level. In this case $\xi_{i}>0\left(\forall k_{x}, k_{y}\right)$ and the actual energy gap $\Delta_{E}^{(n)}$ for the $n$ branch differs from $\left|\Delta_{n}\right|$;

$$
\Delta_{E}^{(n)}=\min _{k_{x}, k_{y}} E_{k_{x}, k_{y}, n}>\left|\Delta_{n}\right| .
$$

Thus, for the total-energy gap $\Delta_{E}$ (as measured in experiments) we have

$$
\Delta_{E}=\min _{n} \Delta_{E}^{(n)},
$$

which is not always the same as $\min _{n}\left|\Delta_{n}\right|$.

Concluding this section, we remark that the NATS criterion based on Eq. (17) was formulated in the absence of a magnetic field and a supercurrent. Its generalization to these more complex cases requires additional investigations. However, there are several particular examples [superconducting 


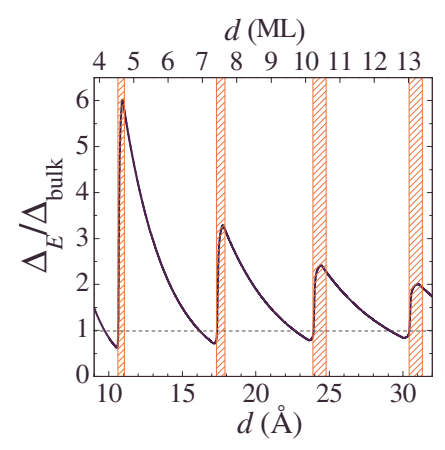

FIG. 1. (Color online) The zero-temperature energy gap $\Delta_{E}$ in units of the bulk gap $\Delta_{\text {bulk }}$ versus the film thickness $d$ of an $\mathrm{Al}$ nanofilm. Sectors where NATS appear are hatched with red lines. The top axis gives the film thickness in ML where $1 \mathrm{ML}$ $=2.338 \AA$ is chosen for $\mathrm{Al}$ grown in the [111] crystalline direction. Note that the values of the top axis are somewhat conditional because of a possible shift up to 1-2 A between the two scales due to charge spillage at the film edges (Ref. 25).

cylindrical nanowires with a parallel magnetic field and superconducting films (wires) with a supercurrent parallel to the film (wire)] when Eq. (17) is also valid in the case of a nonzero magnetic field and supercurrent.

\section{NUMERICAL RESULTS}

As mentioned in Sec. II, the single-electron subbands formed due to the transverse quantization moves in energy when $d$ changes. Therefore, the number of single-electron states in the Debye window strongly varies with the thickness, which gives rise to the quantum-size variations of the superconducting characteristics with profound enhancements at the resonant points (for more detail, see Refs. 17). Such quantum-size oscillations of the energy gap $\Delta_{E}$ of an aluminum nanofilm with uniform thickness $d$ are given in Fig. 1. The data were calculated by numerically solving the $\mathrm{BdG}$ Eqs. (1a) and (1b) at zero temperature for the parameters $\hbar \omega_{D}=32.31 \mathrm{meV}, g N(0)=0.18$, and $E_{F}=0.9 \mathrm{eV}$, where $N(0)$ is the bulk density of states at the Fermi level. Note that the BdG Eqs. (1a) and (1b) are written in the parabolic band approximation and for nanofilms, this implies usage of an effective Fermi level. ${ }^{17}$ We remark that to avoid the parabolic band approximation and usage of the effective Fermi level, one needs to embark on enormously complicated approach accounting for the interplay between the crystal directions, quantum confinement, and superconductivity. The tightbinding approximation can be a reasonable compromise in this situation (see, for instance, Ref. 23). We take also into account that with a decrease in thickness, $E_{F}$ systematically shifts up provided that the mean-electron density stays the same. However, this shift is only significant at $d \lesssim 15 \AA$. For the chosen parameters, the bulk BCS coherence length and energy gap are $\xi_{0}=1.5 \mu \mathrm{m} \gg d$ and $\Delta_{\text {bulk }}=0.25 \mathrm{meV}$, respectively. We remark that our choice of $\mathrm{Al}$ is not critical for the derived conclusions. In particular, the quantum-size oscillations for $\mathrm{Sn}$ and $\mathrm{Pb}$ are less profound by a factor of 2 but the qualitative picture remains the same. In addition, we assume the bulk phonons and do not address any issue con- cerning a thickness-dependent change in the electron-phonon coupling. Such a change (e.g., an increase due to the softening of the phonon spectrum ${ }^{24}$ or a decrease due to charge spillage $^{25}$ or substrate effects ${ }^{2}$ ) is of no importance for our conclusions.

The energy gap in Fig. 1 exhibits a sequence of profound resonant enhancements appearing with the periodicity of about $\lambda_{F} / 2=0.65 \mathrm{~nm}$ (with $\lambda_{F}$ as the Fermi wavelength). The quantum-size oscillations of the superconducting properties with accompanying superconducting resonances are a characteristic feature of high-quality nanosuperconductors. The first predictions about this quantum-size regime dates back to the pioneering paper by Blatt and Thompson. ${ }^{26} \mathrm{Re}$ cently, such oscillations of the critical temperature $T_{c}$ were observed in experiments with atomically uniform singlecrystalline $\mathrm{Pb}$ nanofilms. 2,3 Photoemission electron spectra measured for the same films demonstrated a solid correlation of the critical-temperature oscillations with the variations of the density of states at the Fermi level (see the first paper in Ref. 2). Note that the observed quantum-size oscillations of $T_{c}$ are well reproduced within our numerical solution of the BdG equations. ${ }^{17}$ In addition, it was recently found ${ }^{27}$ that the quantum-size superconducting resonances are responsible for a thickness-dependent increase in the energy gap measured in experiments with $\mathrm{Al}$ polycrystalline nanofilms with strong coupling between the grains. ${ }^{28}$ In these high-quality films, the grain size was shown to have no appreciable effect on the energy gap.

The resonant enhancements in $\Delta_{E}$ are accompanied by an increase in the superconducting order parameter being nonuniform in the transverse direction due to quantum confinement. This results in NATS formation and the sectors, where NATS play a significant role, are highlighted in Fig. 1. The most surprising fact is that (as opposed to recent results on NATS in superconducting nanowires ${ }^{20}$ ) here, such states are only active near the points where the resonances start to develop, i.e., in very small intervals $\Delta d \sim 0.5-0.8 \AA$ separated by steps of about $\lambda_{F} / 2$. The length of such an interval slightly increases from 0.5 up to $0.8 \AA$ while $E_{F}$ approaches its bulk limit. The formation of NATS results in a drop of the ratio of the energy gap (calculated at $T=0$ ) to the critical temperature $T_{c}$ [see Fig. 2(b)]. More precisely, with an increase in $d$, this ratio first exhibits small oscillations about its bulk BCS value of 1.76 [see the inset in Fig. 2(b)]. Then, it drops sharply down with a subsequent relatively slow growth back to 1.76. As seen from the inset in Fig. 2(a), this behavior is accompanied by a local drop in $\Delta_{E}$ as a function of $d$. To go in more detail about the numerical results presented in Fig. 2, the quantities $\Delta_{n}, \Delta_{E}^{(n)}, \Delta_{E}$ (calculated at $T=0$ in units of $\Delta_{\text {bulk }}$ ), and $T_{c}$ (given in units of $T_{c \text {,bulk }}=\Delta_{\text {bulk }} / 1.763 k_{B}$ ) are plotted in Fig. 3 as function of the thickness. The superconducting resonance situated at $d=10.9 \AA$ occurs as a result of the passage of the single-electron subband with $n=1$ through the Fermi surface. So, there are two relevant subbands in this case, i.e., $n=0$ and $n=1$. As the bottom of the single-electron subband with $n=0$ is situated well below the Fermi level, we have $\Delta_{E}^{(0)}=\Delta_{0}$ [see Fig. 3(a)]. This is not always the case for $n=1$ because, to the left of the point $B(d=10.672 \AA)$, the bottom of the corresponding single-electron subband is above the Fermi level. The quasiparticle states with $n=1$ are 


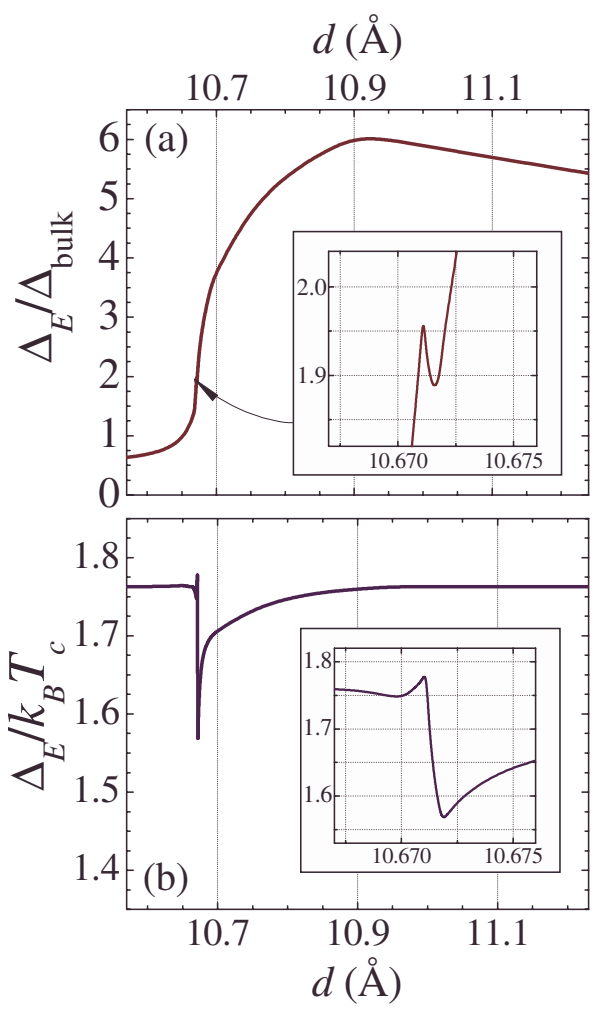

FIG. 2. (Color online) The quantum-size superconducting resonance with the culmination point $d=10.92 \AA$ : (a) the zerotemperature energy gap $\Delta_{E}$ in units of $\Delta_{\text {bulk }}$ as a function of $d$ and (b) the corresponding ratio $\Delta_{E} / k_{B} T_{c}$ versus the film thickness (its bulk limit $\Delta_{\text {bulk }} / k_{B} T_{c \text {,bulk }}=1.763$ ).

NATS because $\Delta_{1}<\Delta_{0}$ (see the criterion for NATS in Sec. II). We remark here that contrary to Anderson's approximate solution, $\Delta_{n}$ cannot be directly calculated from a numerical solution of the BdG equations. However, one can fit the analytical expression given by Eq. (10) to the numerical data for the quasiparticle spectrum. The same result for $\Delta_{n}$ can be derived by calculating the mean value of $\Delta(z)\left(\Delta_{n}\right.$ and $\Delta(z)$ are chosen as real quantities). Thus, to the right of the point $B$, the energy gap $\Delta_{E}$ coincides with $\Delta_{E}^{(1)}=\Delta_{1}$. Between the points $A$ and $B$ the total-energy gap is equal to $\Delta_{E}^{(1)}>\Delta_{1}$. To the left of point $A$ we have $\Delta_{E}=\Delta_{E}^{(0)}=\Delta_{0}$. This explains the local drop of $\Delta_{E}$ in the inset of Fig. 2(a). At the same time $T_{c}$ monotonically increases with $d$ similar to $\Delta_{0}$ and $\Delta_{1}$. Thus, when comparing $\Delta_{E}$ and $T_{c}$ in Fig. 3(b), one can merely understand the features of $\Delta_{E} / k_{B} T_{c}$ given in Fig. 2(b). As follows from our analysis, the drop in $\Delta_{E} / k_{B} T_{c}$ by approximately $10 \%$ in Fig. 2(b), is due to the formation of NATS. When $d$ increases up to $10.92 \AA$ and the corresponding quantum-size resonance fully develops, $\Delta_{1}$ approaches $\Delta_{0}$ and the NATS effect disappears. As a result, $\Delta_{E} / k_{B} T_{c}$ increases back to its bulk limit. Another NATS appear only when the next quantum-size superconducting resonance associated with $n=2$ is brought into play. In this case $\Delta_{0}=\Delta_{1}$ $>\Delta_{2}$ and the quasiparticles with $n=2$ should be interpreted as NATS. Generally, every time the superconducting resonance related to the $n$-subband starts to develop, we have $\Delta_{0}=\Delta_{1}=\ldots=\Delta_{n-1}>\Delta_{n}$. NATS are only active near the point of the resonance buildup and associated with the single-

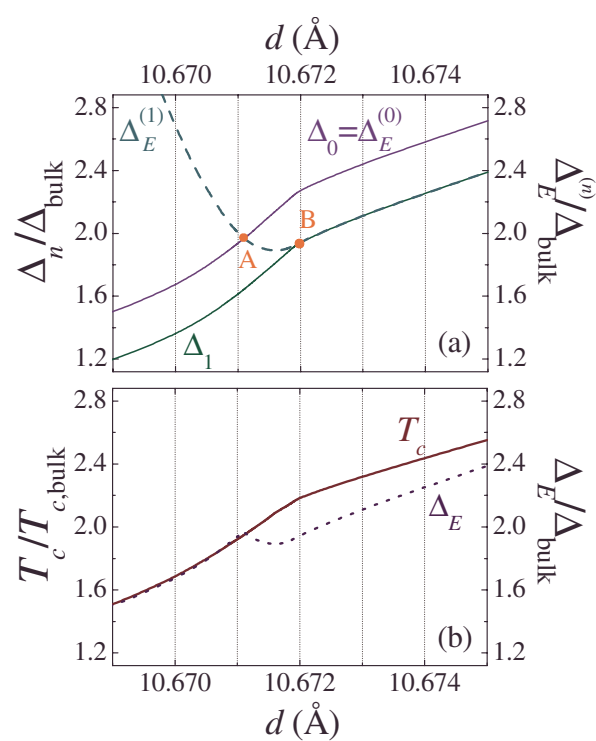

FIG. 3. (Color online) The build-up stage of the superconducting resonance of Fig. 2: (a) $\Delta_{n}$ and $\Delta_{E}^{(n)}$ as functions of the film thickness for $n=0,1$ at $T=0$ and (b) the zero-temperature quasiparticle energy gap $\Delta_{E}$ together with the critical temperature $T_{c}$ versus $d$.

electron subband responsible for the resonance creation. This behavior is very different from the results for nanowires. ${ }^{20}$ In superconducting nanowires, NATS are active from the resonance-culmination point until the next resonance starts to develop. Moreover, the quasiparticle states associated with the single-electron subband responsible for a superconducting resonance are never NATS in the case of nanofilms.

It is of interest to check our conclusions by considering the transverse spatial distribution of the quasiparticles. Let us again take the resonance with the culmination point $d$ $=10.92 \AA$ and the relevant subbands $n=0$ and $n=1$. The particlelike and holelike wave functions are coupled in the presence of superconducting order and, as follows from Anderson's "recipe," they are proportional to one another. It means that the product $u_{i}(\mathbf{r}) v_{i}^{*}(\mathbf{r})$ can provide us with all information about the spatial distribution of quasiparticles. This product does not depend on $k_{x}$ and $k_{y}$ (see Sec. II). So, if we split the order parameter into two parts, i.e., the contributions of the subbands with $n=0$ and $n=1$, each of these parts will be proportional to $u_{n}(z) v_{n}^{*}(z)$. Thus, splitting the order parameter in this way, we can check both the spatial transverse distribution of the corresponding quasiparticles and their input to $\Delta(\mathbf{r})$. In Fig. 4 the contributions of the quasiparticles with $n=0$ and $n=1$ are compared to the order parameter for the two points: (a) $d=10.67 \AA$, the thickness of the minimum in $\Delta_{E} / k_{B} T_{c}$ [see Fig. 2(b)] and (b) $d$ $=11.1 \AA$, where $\Delta_{E} / k_{B} T_{c}$ is back to its bulk value. As seen from Fig. 4(a), the quasiparticles with $n=1$ are remote from the local enhancement of the order parameter in the center of the specimen. Their contribution to $\Delta(z)$ is minor. The states with $n=0$ give the major input to $\Delta(z)$ and, in turn, to the critical temperature. So, $T_{c}$ is controlled by the subband with $n=0$ (having a larger gap $\Delta_{0}$ ), whereas $\Delta_{E}$ is equal to $\Delta_{1}$ and so determined by NATS. This explains the drop in $\Delta_{E} / k_{B} T_{c}$ 


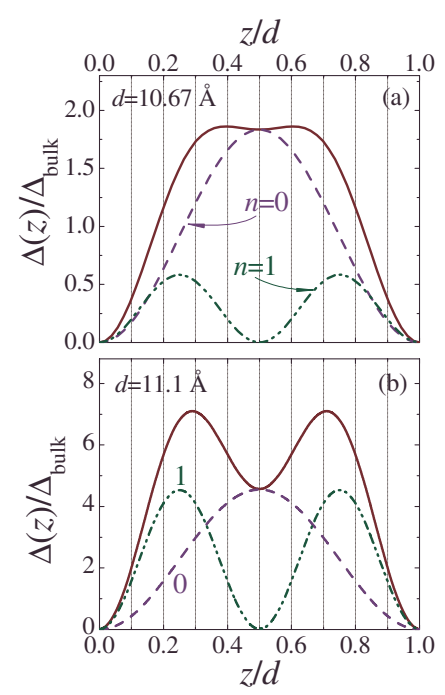

FIG. 4. (Color online) The order parameter (solid curve) as compared to the contributions of the subbands with $n=0$ and 1 to the order parameter (in units of $\Delta_{\text {bulk }}$ ): (a) $d=10.67 \AA$, NATS are present and most active and (b) $d=11.1 \AA$ with no NATS.

by $10 \%$ [it is of the order of $\left(\Delta_{0}-\Delta_{1}\right) / \Delta_{0}$ ]. Now let us turn to Fig. 4(b). Here the superconducting resonance is fully developed and even starts to decay and both the states with $n=0$ and $n=1$ make a significant contribution to the order parameter. Contrary to Fig. 4(a), it is difficult to make a definite conclusion about NATS from Fig. 4(b). However, one can expect that a difference between $\Delta_{0}$ and $\Delta_{1}$ is now less significant. Numerical results give $\Delta_{0}=\Delta_{1}=1.423 \mathrm{meV}$ at $d=11.1 \AA$. For comparison, $\Delta_{0}=1.511 \mathrm{meV}$ and $\Delta_{1}=1.509 \mathrm{meV}$ at $d=10.9 \AA$.

Surprisingly, the single-electron subband that induces a superconducting resonance in films does not make the major contribution to the order parameter (see, for instance, Fig. 4). This is opposed to nanowires where such a subband fully controls the order-parameter (transverse) profile and the superconducting critical temperature. Due to this fact, the transverse spatial distribution of the pair condensate in nanowires is strongly inhomogeneous and changes from resonance to resonance. In contrast, in nanofilms the order parameter is more uniform and its profile does not depend much on the resonance in question. This is why the NATS effect is significantly less pronounced in films.

When increasing $d$, the NATS effect nearly disappears when $d$ approaches $6-7 \mathrm{~nm}$. In Fig. 5 the ratio $\Delta_{E} / k_{B} T_{c}$ is plotted versus the film thickness in the nearest vicinity of the superconducting resonance situated at $d=31.1 \AA$. It is instructive to compare the amplitude of the drop of $\Delta_{E} / k_{B} T_{c}$ in Fig. 5 to that of Fig. 2(a). As seen, the drop is reduced by a factor of 2.5. At $d=7 \mathrm{~nm}$ the ratio $\Delta_{E} / k_{B} T_{c}$ deviates from its bulk value only by $1 \%-2 \%$.

\section{DISCUSSION}

As shown above, NATS are active only in extremely small intervals of thickness $\Delta d \sim 0.1 \lambda_{F} / 2$ occurring with a period of about $\lambda_{F} / 2$ (recall that for our parameters, $\lambda_{F} / 2=6.5 \AA$ ). So, the effect of NATS on the ratio of the

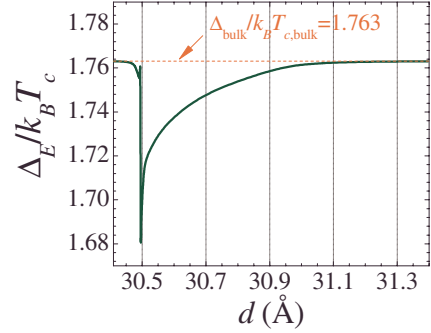

FIG. 5. (Color online) The ratio $\Delta_{E} / K_{B} T_{c}$ versus the thickness for the quantum-size resonance with the culmination point $d=31.1 \AA$.

energy gap to the critical temperature can hardly be observed in metallic superconducting nanofilms. This is in agreement with recent experimental observations ${ }^{3}$ that this ratio in $\mathrm{Pb}$ single-crystalline nanofilms is the same as in bulk.

Nevertheless, we believe that NATS can be investigated in superconducting semiconductors (see, for instance, Refs. 29 and 30 and recent papers on boron-doped diamond ${ }^{31,32}$ and boron-doped silicon ${ }^{33}$ ) where $\lambda_{F}$ is an order of magnitude larger than in metals. Interest in films made of superconducting semiconductors dates back to the paper by Tavger and Demihovskii ${ }^{34}$ (see also Ref. 35). Now this interest has been renewed due to advances in nanofabrication technologies. ${ }^{36}$ One can expect that the low-carrier-density nanosystems are very promising for study of quantum-size oscillations of the superconducting characteristics accompanied by size-dependent resonances. As discussed above, the resonant enhancements in nanofilms appear with a period of about $\lambda_{F} / 2$ when changing the thickness. Therefore, for metallic nanosuperconductors even extremely small size fluctuations of $\sim 0.2-0.5 \mathrm{~nm}$ will smooth out such resonances. This is not the case for nanostructures made of low-carrierdensity superconducting materials, which opens up a unique possibility of tuning the superconducting characteristics by changing the sample dimensions (and the carrier densities). To illustrate our discussion, Fig. 6 shows $\Delta_{E}$ (at $T=0$ ) calculated from the $\mathrm{BdG}$ equations for a superconducting film with the parameters of $\mathrm{SrTiO}_{3}$. This material is good for our illustration because it exhibits superconducting behavior at very low-carrier densities of $10^{18}-10^{19} \mathrm{~cm}^{-3}$ (see Ref. 29). For Fig. 6 we use the following parametric set (Ref. 35):

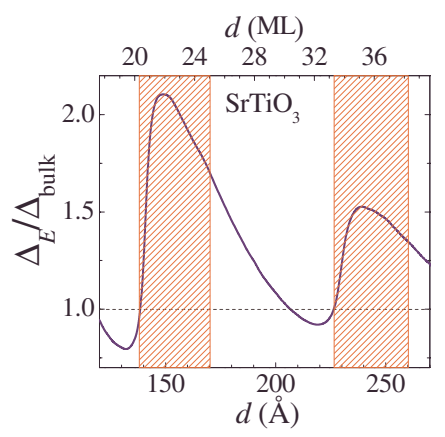

FIG. 6. (Color online) The excitation energy gap $\Delta_{E} / \Delta_{\text {bulk }}$ versus thickness $d$ for nanofilms of $\mathrm{SrTiO}_{3}$. For the top axis we use (Ref. 35) $1 \mathrm{ML}=6.8 \AA$, the domains where NATS appear are hatched in red. 
$\hbar \omega_{D}=49.7 \mathrm{meV}, g N(0)=0.09$ (with the electron density of $5 \times 10^{19} \mathrm{~cm}^{-3}$ ) and $m=5 m_{e}$ for three relevant valleys. This illustrative set is rather simplified, e.g., we do not introduce anisotropic effective masses. In addition, we do not touch the complex interplay of the valley directions with the film orientation. Instead, it is assumed that the energies of electrons in different valleys are equally shifted due to the transverse quantization [this is likely to be the case for (111) films with the lattice constant $6.8 \AA$ in the [111] direction $\left.{ }^{35}\right]$. For the chosen parameters, $\Delta_{\text {bulk }}=0.016 \mathrm{meV}$. It is instructive to compare Fig. 1 with Fig. 6. Now we have $\lambda_{F} / 2=85.6 \AA$ versus $1 \mathrm{ML}=6.8 \AA$ and, as seen from the top axis, NATS are formed in quite reasonable intervals $\Delta d \approx 5 \mathrm{ML}$. The increase in $\Delta d$ (as compared to metallic nanofilms) is due to the two following reasons: (1) $\lambda_{F}$ is significantly larger and, in addition, (2) $\Delta d \sim 0.3 \lambda_{F} / 2$ as opposed to $0.1 \lambda_{F} / 2$ in the metallic case. The second point is due to the fact that we now have $E_{F}<\hbar \omega_{D}$. A drop in $\Delta_{E} / k_{B} T_{c}$ is found to be about $5 \%$ at $d \approx 150 \AA$ (this drop is always in solid correlation with the amplitudes of the resonant enhancements).

Note that single-crystalline films made of boron-doped superconducting diamond are now attainable. ${ }^{36}$ However, we remark that possible strong disorder due to boron impurities can be a serious obstacle for observations of NATS and quantum-size oscillations in this case.

\section{CONCLUSIONS}

Concluding, quantum confinement for the transverse electron motion in high-quality superconducting nanofilms is the major effect governing the superconducting characteristics and resulting in a nonuniform transverse distribution of the superconducting order parameter. Such a nonuniform distribution gives rise to the formation of NATS being a natural nanoscale generalization of the well-known Andreev states. Here we investigated NATS in superconducting nanofilms. Based on Anderson's approximate solution of the BdG equations, we formulated the criterion for NATS formation. Then, by numerically solving the $\mathrm{BdG}$ equations and keeping in mind this criterion, we found that the formation of NATS can reduce the ratio of the energy excitation gap to the critical temperature in high-quality nanofilms by $5 \%-10 \%$. This is less significant as compared to $50 \%$ found in our previous investigation ${ }^{20}$ of NATS in nanowires with thickness of about $2-3 \mathrm{~nm}$. Another issue is that in metallic superconducting nanofilms, NATS can only be formed in extremely narrow intervals of thickness $\Delta d$ $\sim 0.1 \lambda_{F} / 2(\sim 0.1 \mathrm{~nm})$. Thus, we expect that films made of low-carrier-density superconducting materials are more promising for the observations of NATS and other quantumsize superconducting effects.

\section{ACKNOWLEDGMENTS}

This work was supported by the Flemish Science Foundation (FWO-VI) and the Belgian Science Policy (IAP).
${ }^{1}$ http://www.nanowerk.com/spotlight/spotid=4201.php

${ }^{2}$ Y. Guo, Y.-F. Zhang, X.-Y. Bao, T.-Z. Han, Z. Tang, L.-X. Zhang, W.-G. Zhu, E. G. Wang, Q. Niu, Z. Q. Qiu, J.-F. Jia, Z.-X. Zhao, and Q. K. Xue, Science 306, 1915 (2004); X.-Y. Bao, Y.-F. Zhang, Y. Wang, J.-F. Jia, Q.-K. Xue, X. C. Xie, and Z.-X. Zhao, Phys. Rev. Lett. 95, 247005 (2005).

${ }^{3}$ D. Eom, S. Qin, M.-Y. Chou, and C. K. Shih, Phys. Rev. Lett. 96, 027005 (2006).

${ }^{4}$ M. M. Özer, J. R. Thompson, and H. H. Weitering, Nat. Phys. 2, 173 (2006); M. M. Özer, J. R. Thompson, and H. H. Weitering, Phys. Rev. B 74, 235427 (2006).

${ }^{5}$ M. L. Tian, J. G. Wang, J. S. Kurtz, Y. Liu, M. H. W. Chan, T. S. Mayer, and T. E. Mallouk, Phys. Rev. B 71, 104521 (2005).

${ }^{6}$ L. Jankovič, D. Gournis, P. N. Trikalitis, I. Arfaoui, T. Cren, P. Rudolf, M.-H. Sage, T. T. M. Palstra, B. Kooi, J. De Hosson, M. A. Karakassides, K. Dimos, A. Moukarika, and T. Bakas, Nano Lett. 6, 1131 (2006).

${ }^{7}$ M. Zgirski, K.-P. Riikonen, V. Touboltsev, and K. Arutyunov, Nano Lett. 5, 1029 (2005).

${ }^{8}$ F. Altomare, A. M. Chang, M. R. Melloch, Y. Hong, and C. W. Tu, Phys. Rev. Lett. 97, 017001 (2006).

${ }^{9}$ P. W. Anderson, J. Phys. Chem. Solids 11, 26 (1959).

${ }^{10}$ A. A. Abrikosov and L. P. Gorkov, Zh. Eksp. Teor. Fiz. 35, 1558 (1958) [Sov. Phys. JETP 8, 1090 (1959)]; Zh. Eksp. Teor. Fiz. 36, 319 (1959) [Sov. Phys. JETP 9, 220 (1959)].

${ }^{11}$ B. L. Altshuler, A. G. Aronov, and P. A. Lee, Phys. Rev. Lett. 44, 1288 (1980).
${ }^{12}$ B. L. Altshuler, JETP Lett. 41, 648 (1985); P. A. Lee and A. D. Stone, Phys. Rev. Lett. 55, 1622 (1985).

${ }^{13}$ M. A. Skvortsov and M. V. Feigel'man, Phys. Rev. Lett. 95, 057002 (2005).

${ }^{14}$ Y. Oreg and A. M. Finkelstein, Phys. Rev. Lett. 83, 191 (1999).

${ }^{15}$ N. N. Bogoliubov, Sov. Phys. JETP 7, 41 (1958).

${ }^{16}$ L. P. Gor'kov, Sov. Phys. JETP 7, 505 (1958).

${ }^{17}$ A. A. Shanenko, M. D. Croitoru, and F. M. Peeters, Europhys. Lett. 76, 498 (2006); Phys. Rev. B 75, 014519 (2007); M. D. Croitoru, A. A. Shanenko, and F. M. Peeters, ibid. 76, 024511 (2007).

${ }^{18}$ A. F. Andreev, Sov. Phys. JETP 22, 455 (1966).

${ }^{19}$ C. Caroli, P. G. de Gennes, and J. Matricon, Phys. Lett. 9, 307 (1964).

${ }^{20}$ A. A. Shanenko, M. D. Croitoru, R. G. Mints, and F. M. Peeters, Phys. Rev. Lett. 99, 067007 (2007).

${ }^{21}$ P. G. de Gennes, Superconductivity of Metals and Alloys (Benjamin, New York, 1966).

${ }^{22}$ E. H. Hwang, S. Das Sarma, and M. A. Stroscio, Phys. Rev. B 61, 8659 (2000); B. Chen, Z. Zhu, and X. C. Xie, ibid. 74, 132504 (2006).

${ }^{23}$ Y. S. Barash and P. I. Nagornykh, JETP Lett. 83, 376 (2006).

${ }^{24}$ D. G. Naugle, J. W. Baker, and R. E. Allen, Phys. Rev. B 7, 3028 (1973).

${ }^{25}$ M. Yu, M. Strongin, and A. Paskin, Phys. Rev. B 14, 996 (1976).

${ }^{26}$ J. M. Blatt and C. J. Thompson, Phys. Rev. Lett. 10, 332 (1963).

${ }^{27}$ F. M. Peeters, M. D. Croitoru, and A. A. Shanenko, Physica C 
468, 326 (2008).

${ }^{28}$ N. A. Court, A. J. Ferguson, and R. G. Clark, Supercond. Sci. Technol. 21, 015013 (2008).

${ }^{29}$ J. F. Schooley, W. R. Hosler, and M. L. Cohen, Phys. Rev. Lett. 12, 474 (1964); J. F. Schooley, W. R. Hosler, E. Ambler, J. H. Becker, M. L. Cohen, and C. S. Koonce, ibid. 14, 305 (1965).

${ }^{30}$ R. A. Hein, J. W. Gibson, R. Mazelsky, R. C. Miller, and J. K. Hulm, Phys. Rev. Lett. 12, 320 (1964).

${ }^{31}$ E. A. Ekimov, V. A. Sidorov, E. D. Bauer, N. N. Mel'nik, N. J. Curro, J. D. Thompson, and S. M. Stishov, Nature (London) 428, 542 (2004).

${ }^{32}$ Y. Takano, M. Nagao, I. Sakaguchi, M. Tachiki, T. Hatano, K.
Kobayashi, H. Umezawa, and H. Kawarada, Appl. Phys. Lett. 85, 2851 (2004).

${ }^{33}$ E. Bustarret, C. Marcenat, P. Achatz, J. Kačmarčik, F. Levy, A. Huxley, L. Ortega, E. Bourgeois, X. Blase, D. Debarre, and J. Boulmer, Nature (London) 444, 427 (2006).

${ }^{34}$ B. A. Tavger and V. Yu. Demihovskii, Sov. Phys. JETP 21, 494 (1965).

${ }^{35}$ D. M. Eagles, Phys. Rev. 164, 489 (1967).

${ }^{36}$ E. Bustarret, J. Kačmarčik, C. Marcenat, E. Gheeraert, C. Cytermann, J. Marcus, and T. Klein, Phys. Rev. Lett. 93, 237005 (2004). 